\title{
The Three-Factor Eating Questionnaire and BMI in adolescents: results from the Québec Family Study
}

\author{
A. R. Gallant ${ }^{1}$, A. Tremblay $^{1}$, L. Pérusse ${ }^{1}$, C. Bouchard ${ }^{2}$, J.-P. Després ${ }^{1,3}$ and V. Drapeau ${ }^{4 *}$ \\ ${ }^{1}$ Division of Kinesiology, Department of Preventive Medicine, Université Laval, Québec, QC, Canada G1V OA6 \\ ${ }^{2}$ Pennington Biomedical Research Center, Baton Rouge, LA, USA \\ ${ }^{3}$ Quebec Heart and Lung Institute, Université Laval, Québec, QC, Canada \\ ${ }^{4}$ Department of Physical Education (bureau 2214), Université Laval, 2300 rue de la Terrasse, PEPS, Québec, QC, Canada GIV OA6
}

(Received 10 December 2009 - Revised 16 March 2010 - Accepted 30 March 2010 - First published online 7 May 2010)

Eating behaviour traits are associated with body weight variations in adults. The Three-Factor Eating Questionnaire (TFEQ) measures cognitive restraint, disinhibition and hunger, as well as their corresponding subscales, e.g. rigid and flexible control. The TFEQ has not been widely used in adolescents to investigate eating behaviour traits associated with body weight. The aim of the present study was to assess whether eating behaviour traits were associated with BMI in male and female adolescents. Sixty adolescents (thirty females and thirty males; mean age $15 \cdot 0$ (SD 2.4) years) from the Québec Family Study completed the TFEQ and 3d dietary records. There were no sex differences in the TFEQ scores. Rigid control, disinhibition and emotional susceptibility (to overeat) were positively related to BMI $z$-scores for the entire sample $(r 0.3, P<0 \cdot 05)$. There was a positive relationship between BMI $z$-scores and rigid control $(r 0.39, P<0 \cdot 05)$ in females, while BMI $z$-scores were positively related to emotional susceptibility $(r 0.42, P<0.02)$ and disinhibition $(r 0.41, P<0.03)$ in males. Adolescents characterised by both high disinhibition and high rigid control had significantly higher BMI $z$-scores than those by both low disinhibition and low rigid control. There were no significant differences in BMI $z$-scores between the flexible control categories. Dietary macronutrient content was not consistently related to eating behaviour traits. These results show that the eating behaviour traits of disinhibition and rigid control are independently related to BMI $z$-scores in this group of adolescents.

Eating behaviour traits: Adolescents: Restraint: Disinhibition

The obesity pandemic is sweeping every age group. Children and adolescents are increasingly heavier than those of previous generations. In $1978,3 \%$ of the Canadian adolescents between the ages of 12 and 17 years were obese. The prevalence had increased to $9 \%$ by 2004, the largest increase of all the Canadian age groups ${ }^{(1)}$. The causes of obesity are numerous, and include environmental, cultural, familial, behavioural, metabolic and genetic factors. In the last 30 years, research on behavioural issues associated with obesity has expanded; yet, many questions remain unanswered and most conclusions are only tentative.

A number of research questionnaires are used to assess eating behaviour traits. One of the most widely used is the Three-Factor Eating Questionnaire (TFEQ) ${ }^{(2)}$ that measures cognitive restraint, disinhibition and hunger in adults. In 1991, Westenhoefer ${ }^{(3)}$ suggested that cognitive restraint, as measured by the TFEQ, could be divided into two subscales depending on its relationships with BMI and disinhibition: flexible and rigid control. The former, a more relaxed version of restraint, is associated with both low disinhibition and low BMI. The latter is a more severe restrictive state, and is associated with both high disinhibition and high $\mathrm{BMI}^{(4)}$. In addition to the control subscales, Bond et al. ${ }^{(5)}$ expanded on the three main categories by defining more specific subscales for each behaviour trait, e.g. strategic dieting behaviour, emotional susceptibility and avoidance of fattening foods. These TFEQ subscales have been measured in adults ${ }^{(4,6-1)}$; however, to the best of our knowledge, they have never been assessed in adolescents.

The TFEQ has been shown to measure cognitive restraint and disinhibition independently ${ }^{(10,12)}$; yet, these behaviours work in combination as well. Studies have shown that high susceptibility to overeat (disinhibition) combined with low restriction is associated with higher body weight ${ }^{(8,13-15)}$. These interactions have been studied in women using the TFEQ control subscales ${ }^{(10)}$, and in adolescent females, using the Dutch Eating Behaviour Questionnaire ${ }^{(12)}$, with varying results concerning body weight. Thus, eating behaviour traits in adolescents have not been largely studied. The aim of the present study was to assess eating behaviours and their associations with body weight in adolescents.

\section{Methods}

The volunteers for the present study participated in the Québec Family Study, a prospective study which was started in 1978 
and was continued until 2001 (phase 1, 1978-82; phase 2, 1989-95; phase 3, 1997-2001). All methods relevant to the present report are described elsewhere ${ }^{(11)}$. Sixty adolescents completed the TFEQ at the end of phase 2. Self-reported, $3 \mathrm{~d}$ dietary records ${ }^{(16)}$ (two weekdays and one weekend day) were collected, and the Canadian Nutrient File ${ }^{(17)}$ was used to estimate energy intake and diet macronutrient content. Body weight and height were measured using standardised laboratory methods. In addition to the original fifty-one-question TFEQ, subscales for disinhibition, hunger and cognitive restraint ${ }^{(5)}$, as well as the rigid and flexible control subscales $^{(3)}$, were also measured. Lean and overweight/obese categories were determined by obesity cutoffs suggested by Cole et $a l .^{(18)}$ The adolescents were grouped into four categories defined by their levels of restraint (cognitive restraint, flexible control and rigid control) and disinhibition: (1) high disinhibition-high restraint; (2) high disinhibitionlow restraint; (3) low disinhibition-high restraint (4) low disinhibition-low restraint. High and low scores were defined as being above or below the group median with equal group sizes. We selected this method of classification because there are currently no TFEQ score norms for adolescents by which to classify them.

Statistical analyses were performed using JMP 7.0 Statistical Software from Statistical Analysis Systems (SAS Institute, Cary, NC, USA). Means, standard error and interquartile ranges were calculated, and Student's $t$ tests were used to test for sex and weight category differences in mean TFEQ values with the significance level set at $P \leq 0 \cdot 05$. Pearson's correlation coefficient was used to quantify linear relationships. When appropriate, correlations were adjusted for confounding factors such as age and BMI (reported energy intake). As the median split method has been criticised $^{(19)}$, full-factorial multiple regression analysis using the continuous variables restraint and disinhibition was also used to test for variable interaction. An ANOVA was used to compare BMI $z$-scores between various TFEQ categories. When the ANOVA was significant, a post hoc TukeyKramer test was used to detect which conditions were statistically different from each other. To account for the variation in growth rate observed at adolescence, values were transformed into BMI $z$-scores using the US Centre for Disease Control and Prevention 2000 growth charts for children, aged 2-20 years ${ }^{(20)}$. The present study was approved by the Medical Ethics Committee of Université Laval.

\section{Results}

Characteristics of the study population are given in Table 1. There were no significant sex differences either in physical characteristics or in mean TFEQ scores, except for avoidance of fattening foods which was higher in females than in males $(P<0 \cdot 05)$. Rigid control, disinhibition and emotional susceptibility to overeat were all positively related to BMI $z$-scores (Table 2). Among females, but not among males, there was a positive relationship between rigid control and BMI $z$-scores $(P \leq 0 \cdot 04)$. Among males, but not among females, emotional susceptibility and disinhibition were significantly correlated with BMI $z$-scores $(P \leq 0.02$ and $0 \cdot 03$, respectively). Mean values of rigid control, disinhibition and the disinhibition subscales were all higher among the
Table 1. Physical characteristics of the study population by sex (Mean values and interquartile ranges (IQR))

\begin{tabular}{|c|c|c|c|c|}
\hline \multirow{2}{*}{$\begin{array}{l}\text { Physical } \\
\text { characteristics }\end{array}$} & \multicolumn{2}{|c|}{ Females (n 30) } & \multicolumn{2}{|c|}{ Males (n 30) } \\
\hline & Mean & IQR & Mean & IQR \\
\hline Age (years) & $15 \cdot 6$ & $13 \cdot 7-17 \cdot 7$ & 14.4 & $12 \cdot 7-16 \cdot 3$ \\
\hline Weight (kg) & $65 \cdot 2$ & $49 \cdot 6-84 \cdot 4$ & $74 \cdot 8$ & $49.5-90 \cdot 9$ \\
\hline Height (cm) & $161 \cdot 1$ & $155 \cdot 8-170 \cdot 4$ & $166 \cdot 2$ & $152 \cdot 7-175 \cdot 9$ \\
\hline BMI $\left(\mathrm{kg} / \mathrm{m}^{2}\right)$ & $25 \cdot 7$ & $20 \cdot 3-28 \cdot 4$ & $26 \cdot 1$ & $19 \cdot 8-30 \cdot 8$ \\
\hline
\end{tabular}

overweight/obese adolescents (Table 3). In the total sample, rigid and flexible control were positively correlated after adjusting for age and BMI $\left(r^{2} 0 \cdot 11, P<0 \cdot 01\right)$.

The adolescents were grouped into four categories according to their levels and type of restriction (flexible and rigid) and disinhibition. Mean TFEQ scores were all significantly different between low and high categories $(P<0.05$; Table 4). Sex distributions within the TFEQ categories were not significantly different, and for this reason, we did not separate the categories by sex. Full-factorial multiple regression analysis revealed no significant effect of the interaction between disinhibition and cognitive restraint, flexible control or rigid control on BMI $z$-scores (data not shown). However, adolescents characterised by high cognitive restraint-high disinhibition had significantly higher BMI $z$-scores than those characterised by low disinhibition-low cognitive restraint (Fig. 1(A)), as did those characterised by high disinhibitionhigh rigid control when compared with the group characterised by low disinhibition-low rigid control (Fig. 1(B)). There were no significant differences between BMI $z$-scores for the flexible control categories (Fig. 1(C)).

Hunger was positively correlated to self-reported energy intake for the entire sample $\left(r^{2} 0.1, P \leq 0 \cdot 02\right)$. No other TFEQ scales were related to either energy intake or macronutrient content estimated from the dietary records.

\section{Discussion}

To our knowledge, the present study was the first to assess the TFEQ, and its subscales, on an adolescent population of both sexes. The results showed that the rigid control trait was positively related to BMI $z$-scores in females, while emotional susceptibility and disinhibition were associated with BMI $z$-scores in males. In addition to individual TFEQ associations with body weight, eating behaviour categories were also associated with BMI $z$-scores. When the cognitive restraint scale is divided into the restraint subscales (rigid and flexible), the deleterious effect of rigid control on body weight is observed. Although there was no interaction effect of rigid control and disinhibition on BMI $z$-scores, those with both high restraint and high disinhibition had significantly higher BMI $z$-scores than those with low eating behaviour scores, with the negative effect deriving from high rigid control. These behaviours had significant and independent effects on BMI $z$-scores, and a trend towards an additive effect was visible.

Disinhibition, or overeating, is known to be associated with increased body weight ${ }^{(21)}$, whereas the relationship between 
Table 2. Correlations between eating behaviours and BMI z-scores* in adolescents

\begin{tabular}{|c|c|c|c|c|c|c|}
\hline \multirow[b]{3}{*}{ TFEQ scores } & \multicolumn{6}{|c|}{ BMI z-scores } \\
\hline & \multicolumn{2}{|c|}{ Total $(n 60)$} & \multicolumn{2}{|c|}{ Females ( $n 30)$} & \multicolumn{2}{|c|}{ Males $(n 30)$} \\
\hline & $r^{2}$ & $P$ & $r^{2}$ & $P$ & $r^{2}$ & $P$ \\
\hline Rigid control & $0.09(+)$ & 0.02 & $0.15(+)$ & 0.04 & $0.06(+)$ & 0.18 \\
\hline Disinhibition & $0.09(+)$ & 0.02 & $0.07(+)$ & 0.17 & $0.17(+)$ & 0.03 \\
\hline Emotional susceptibility & $0.08(+)$ & 0.03 & $0.07(+)$ & 0.16 & $0.18(+)$ & 0.02 \\
\hline
\end{tabular}

TFEQ, Three-Factor Eating Questionnaire.

${ }^{*} \mathrm{BMI} Z$-scores were calculated from the Center for Disease Control and Prevention 2000 growth charts.

body weight and dietary restraint varies in adults; some show an inverse relationship ${ }^{(9,10,14)}$, while others show no relation$\operatorname{ship}^{(15,22-24)}$. A positive relationship between restrained eating and body weight has been found among adolescents $^{(25,26)}$ and children ${ }^{(27)}$. In the present study, there was no linear relationship between cognitive restraint per se and BMI $z$-scores, but the heavier the adolescents were, the higher their rigid control towards eating was, a result concordant with previous findings in adults $(4,6,7,11,22,28)$. The conflicting results of dietary restraint in the literature coupled with data emerging regarding rigid dietary control show the importance of using the restraint subscales. In the present study, flexible control did not seem to affect body weight as it is not related to BMI $z$-scores.

In accordance with the results obtained in adult men ${ }^{(11)}$, there was a positive relationship between disinhibition and BMI $z$-scores among adolescent males, a relationship not found among females in the present study. Male adolescent eating behaviour studies are rare. One study has shown that male adolescents exhibit higher uncontrolled eating, a derivative of the disinhibition measure in a revised TFEQ (TFEQ-R18) $^{(26)}$, and another study has shown higher external hunger, which is eating in the absence of hunger according to the Dutch Eating Behaviour Questionnaire ${ }^{(29)}$. Not only was disinhibition related to body weight in the present study, but the male adolescents had higher mean disinhibition scores when compared with adult norms ${ }^{(30)}$. Restrained eating seems to be more prevalent in adolescent females ${ }^{(25,26,29)}$, whereas overeating may play an important role in both sexes. Accordingly, a large adolescent study showed that $7.8 \%$ of boys and $17.3 \%$ of girls reported objective overeating, revealing its effects on both sexes ${ }^{(31)}$. A recent review $^{(21)}$ has confirmed the poor health associations of disinhibited eating, and stressed the importance of including young males in eating behaviour/disorder research as the problem is now being observed in young males ${ }^{(32)}$.

Analyses by high and low eating behaviour categories revealed that having both high disinhibition and high restraint, specifically high rigid control, were related to higher BMI $z$-scores. The individuals in the high disinhibition-high rigid control category may be characterised as being unsuccessful in their restrained attempts, which could explain their increased body weight. The fundamental purpose of the rigid control subscale was to show that some individuals are so severely controlled that this behaviour is unable to be maintained, and uncontrolled eating episodes occur ${ }^{(3)}$. It was shown that young females with high dietary restraint in combination with other eating behaviours, specifically disinhibition, were more likely to fail at their restrained efforts and had more eating disorder symptoms, such as binging ${ }^{(12)}$. Disinhibition and rigid control towards eating are the strongest distinguishing factors for obesity in this group of adolescents, since only those with both high disinhibition and high rigid control had significantly higher BMI $z$-scores than those with low levels

Table 3. Three-Factor Eating Questionnaire (TFEQ) scores with corresponding subscales for adolescents (Mean values and interquartile ranges (IQR))

\begin{tabular}{|c|c|c|c|c|c|c|}
\hline \multirow[b]{2}{*}{ Eating behaviours } & \multicolumn{2}{|c|}{ Total $(n 60)$} & \multicolumn{2}{|c|}{ Lean $(n 27)$} & \multicolumn{2}{|c|}{ Overweight/obese ( $n$ 33) } \\
\hline & Mean & IQR & Mean & IQR & Mean & IQR \\
\hline Cognitive dietary restraint & $5 \cdot 1$ & $2 \cdot 0-7 \cdot 0$ & $4 \cdot 1$ & $2 \cdot 0-6 \cdot 0$ & $5 \cdot 9$ & $3.0-8.5$ \\
\hline Flexible control & 1.9 & $1.0-3.0$ & 1.8 & $1.0-3.0$ & $2 \cdot 0$ & $1.0-3.0$ \\
\hline Rigid control & $1 \cdot 0$ & $0.0-2.0$ & 0.52 & $0.0-1 \cdot 0$ & $1.4^{\star *}$ & $0 \cdot 0-2 \cdot 0$ \\
\hline Attitude to self-regulation & 1.6 & $1 \cdot 0-2 \cdot 0$ & 1.4 & $1 \cdot 0-2 \cdot 0$ & 1.8 & $1 \cdot 0-3 \cdot 0$ \\
\hline Strategic dieting behaviour & 0.58 & $0.0-1.0$ & 0.41 & $0.0-1.0$ & 0.72 & $0 \cdot 0-1 \cdot 0$ \\
\hline Avoidance of fattening foods & $1 \cdot 3$ & $1 \cdot 0-2 \cdot 0$ & $1 \cdot 1$ & $0 \cdot 0-2 \cdot 0$ & 1.4 & $1 \cdot 0-2 \cdot 0$ \\
\hline Disinhibition & $6 \cdot 1$ & $3 \cdot 0-9 \cdot 0$ & 4.7 & $2 \cdot 0-6 \cdot 0$ & $7 \cdot 3^{\star \star}$ & $3.5-10 \cdot 0$ \\
\hline Habitual susceptibility & 0.95 & $0.0-1.2$ & 0.60 & $0.0-1.0$ & $1 \cdot 2^{*}$ & $0.0-2 \cdot 0$ \\
\hline Emotional susceptibility & $1 \cdot 25$ & $0.0-3.0$ & 0.78 & $0.0-1.0$ & $1 \cdot 6^{\star \star}$ & $0.0-3.0$ \\
\hline Situational susceptibility & $2 \cdot 1$ & $1 \cdot 0-3 \cdot 0$ & 1.6 & $0.0-3.0$ & $2 \cdot 5^{\star}$ & $1 \cdot 0-4 \cdot 0$ \\
\hline Hunger & 6.6 & $4 \cdot 3-9 \cdot 0$ & 6.9 & $5 \cdot 0-10 \cdot 0$ & $6 \cdot 4$ & $4.0-9.0$ \\
\hline Internal locus for hunger & $2 \cdot 9$ & $1.0-5.0$ & 3.3 & $1 \cdot 0-5 \cdot 0$ & $2 \cdot 8$ & $1 \cdot 0-5 \cdot 0$ \\
\hline External locus for hunger & $2 \cdot 6$ & $1.0-4.0$ & $2 \cdot 7$ & $1 \cdot 0-4 \cdot 0$ & $2 \cdot 4$ & $1 \cdot 0-4 \cdot 0$ \\
\hline
\end{tabular}

Mean values were significantly different from those of lean adolescents: ${ }^{\star} P<0.05,{ }^{\star \star} P<0.01$. 
Table 4. Three-Factor Eating Questionnaire (TFEQ) scores for high and low categories of cognitive restraint, its subscales and disinhibition

(Mean values and interquartile ranges (IQR))

\begin{tabular}{|c|c|c|c|c|c|c|}
\hline \multirow[b]{2}{*}{ TFEQ classification } & \multicolumn{2}{|c|}{ Total $(n 60)$} & \multicolumn{2}{|c|}{ Females $(n 30)$} & \multicolumn{2}{|c|}{ Males ( $n$ 30) } \\
\hline & Mean & IQR & Mean & IQR & Mean & IQR \\
\hline \multicolumn{7}{|l|}{ Cognitive restraint } \\
\hline $\mathrm{High}^{\star}$ & 7.9 & $6 \cdot 0-9 \cdot 0$ & 8.0 & $6.0-9.5$ & $7 \cdot 8$ & $5 \cdot 3-9 \cdot 0$ \\
\hline Low $^{\star}$ & $2 \cdot 3$ & $1 \cdot 0-3 \cdot 0$ & $2 \cdot 9$ & $2 \cdot 0-4 \cdot 0$ & 1.9 & $1 \cdot 0-3 \cdot 0$ \\
\hline \multicolumn{7}{|l|}{ Flexible control } \\
\hline High* & 3.0 & $2 \cdot 0-3 \cdot 6$ & 3.0 & $2 \cdot 0-4 \cdot 0$ & 3.0 & $2 \cdot 0-3.5$ \\
\hline Low $^{*}$ & 0.8 & $0.0-1.9$ & 0.8 & $1 \cdot 0-1 \cdot 0$ & 0.8 & $0.0-1.0$ \\
\hline \multicolumn{7}{|l|}{ Rigid control } \\
\hline $\mathrm{High}^{*}$ & 1.9 & $1 \cdot 0-2 \cdot 3$ & 1.9 & $1 \cdot 0-2 \cdot 3$ & 1.8 & $1 \cdot 0-2 \cdot 8$ \\
\hline Low $^{*}$ & 0.13 & $0.0-0.0$ & 0.1 & $0.0-0.0$ & 0.2 & $0.0-0.0$ \\
\hline \multicolumn{7}{|l|}{ Disinhibition } \\
\hline High $^{*}$ & $9 \cdot 1$ & $7 \cdot 0-10 \cdot 8$ & $9 \cdot 4$ & $7 \cdot 8-11 \cdot 3$ & 8.7 & $7 \cdot 0-10 \cdot 0$ \\
\hline Low* & 3.2 & $2 \cdot 0-4 \cdot 3$ & $2 \cdot 8$ & $2 \cdot 0-3.8$ & 3.4 & $2 \cdot 0-5 \cdot 0$ \\
\hline
\end{tabular}

* Mean values were significantly different from each other for all high-low pairs $(P<0.05)$.
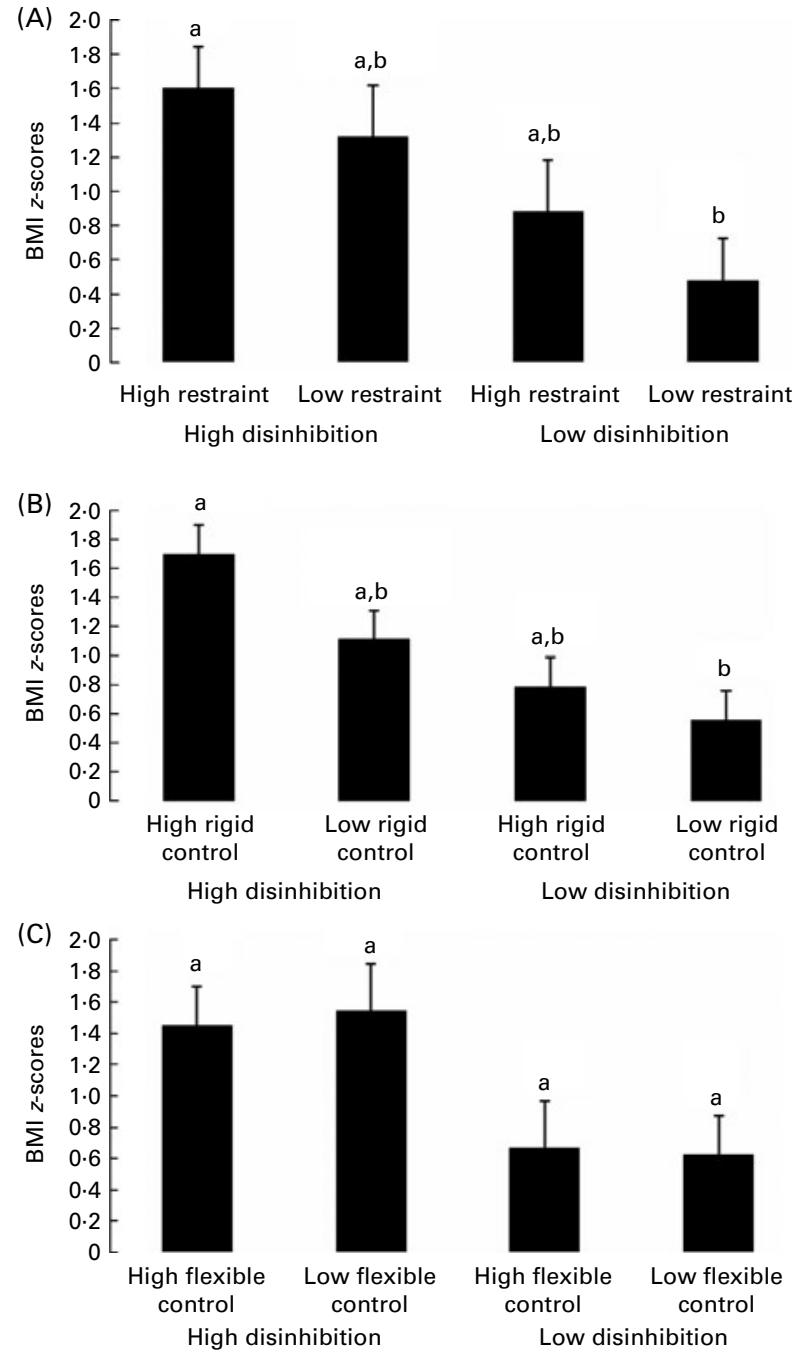

Fig. 1. BMI $z$-scores for (A) cognitive restraint, $(B)$ rigid control and (C) flexible control-disinhibition eating behaviour categories for male and female adolescents. Data are represented as means with their standard errors. Mean values were significantly different as per one-way ANOVA $(P \leq 0.05) .{ }^{a, b}$ Mean values with unlike letters were significantly different from each other as per post hoc Tukey-Kramer test $(P \leq 0.05)$. of both behaviours. As there were no significant differences between the flexible control categories, flexible control may have a moderating effect on BMI. This is not surprising as flexible control is known to be a more successful approach to weight loss ${ }^{(4)}$. Several studies have found that restraint has a moderating effect on the interaction between disinhibition and body weight ${ }^{(14,15,23)}$; yet, these studies did not use the restraint subscales. Using the restraint subscales may shed more light on the potential moderating effect of restraint.

Various factors could explain the trends observed herein. The categories were formed on the basis of the study sample means. The lack of TFEQ research on adolescents limits comparisons. However, when comparing these values with US adult TFEQ norms ${ }^{(30)}$ and with results obtained in children (12 years $)^{(27)}$, overall high disinhibition scores among these adolescents were observed, specifically among the males. In contrast, cognitive restraint, rigid control and flexible control scores were all lower in this population than in adults ${ }^{(4,6,11)}$ and children ${ }^{(27)}$. Thus, the high disinhibition scores coupled with the low restraint scores may be largely responsible for the lack of significant differences between restraint and disinhibition categories.

In the present study, hunger was the only TFEQ score which was associated with absolute energy intake. One study also reported a similar lack of associations between TFEQ scores and energy intake ${ }^{(33)}$; however, most studies have reported these associations ${ }^{(13,24,25,34)}$. Discrepancies could be explained in part by under-reporting in adolescents, which has been found to be as much as $34 \%^{(35)}$. BMI values ${ }^{(36)}$ and eating restraint scores (for females) are also positively correlated with under-reporting in dietary analysis in adolescent populations $^{(37,38)}$. The lack of associations between the TFEQ scores and self-reported energy intake could in part be explained by these phenomena; yet, further research is needed in this area.

There are other limitations with the present study. The lack of high and low norms for eating behaviours resulted in the use of group-specific separations, and this should be taken into account when interpreting the present results. A larger sample size would be needed to generate more definitive tests of the trends observed herein. Finally, because of the 
cross-sectional nature of these associations, it is impossible to establish cause and effect relationships.

In conclusion, eating behaviours in adolescents are associated with body weight, similar to what is observed in adult studies. The TFEQ is able to identify relevant eating behaviour traits associated with higher BMI in this mixedsex age group. Disinhibition and rigid control should be targeted in adolescents in order to characterise youth at risk for obesity, and to implement proper weight control strategies or to predict success or failure in weight-loss participants.

\section{Acknowledgements}

The present work was funded by the Canadian Institutes of Health Research (MCG-15187). A. R. G. is a recipient of the Richard J. Schmeelk Fellowship. The authors specially thank G. Fournier, L. Allard, M. Chagnon and C. Leblanc for their contributions to the recruitment and data collection of the Québec Family Study. Québec Family Study was supported over the years by multiple grants from the Medical Research Council of Canada and the Canadian Institutes for Health Research (PG-11811, MT-13960 and GR-15187) as well as other agencies. There are no conflicts of interest for the present study. The authors' contributions are as follows: A. R. G. carried out the literature review, data extraction and interpretation of the data, and drafted the manuscript. V. D. participated in the conceptualisation, data extraction and interpretation of the data, and helped to draft the manuscript. A. T., L. P., C. B. and J.-P. D. contributed to the conceptualisation and data interpretation. All the authors revised the manuscript.

\section{References}

1. Canada Statistics (2004) Canadian Community Health Survey. http://www.statcan.gc.ca/dailyquotidien/050706/dq050706a-eng. htm

2. Stunkard AJ \& Messick S (1985) The Three-Factor Eating Questionnaire to measure dietary restraint, disinhibition and hunger. J Psychosom Res 29, 71-83.

3. Westenhoefer J (1991) Dietary restraint and disinhibition: is restraint a homogeneous construct? Appetite 16, 45-55.

4. Westenhoefer J, Stunkard AJ \& Pudel V (1999) Validation of the flexible and rigid control dimensions of dietary restraint. Int J Eat Disord 26, 53-64.

5. Bond MJ, McDowell AJ \& Wilkinson JY (2001) The measurement of dietary restraint, disinhibition and hunger: an examination of the factor structure of the Three-Factor Eating Questionnaire (TFEQ). Int J Obes Relat Metab Disord 25, 900-906.

6. Drapeau V, Provencher V, Lemieux S, et al. (2003) Do 6-y changes in eating behaviours predict changes in body weight? Results from the Québec Family Study. Int $J$ Obes Relat Metab Disord 27, 808-814.

7. Timko CA \& Perone J (2005) Rigid and flexible control of eating behaviour in a college population. Eat Behav 6, $119-125$.

8. Westenhoefer J, Broeckmann P, Munch AK, et al. (1994) Cognitive control of eating behaviour and the disinhibition effect. Appetite 23, 27-41.

9. Shearin EN, Russ MJ, Hull JW, et al. (1994) Construct validity of the Three-Factor Eating Questionnaire: flexible and rigid control subscales. Int J Eat Disord 16, 187-198.
10. Williamson DA, Lawson OJ, Brooks ER, et al. (1995) Association of body mass with dietary restraint and disinhibition. Appetite 25, 31-41.

11. Provencher V, Drapeau V, Tremblay A, et al. (2003) Eating behaviours and indexes of body composition in men and women from the Québec family study. Obes Res 11, 783-792.

12. Van Strien T (1997) The concurrent validity of a classification of dieters with low versus high susceptibility toward failure of restraint. Addict Behav 22, 587-597.

13. Westenhoefer J, Pudel V \& Maus N (1990) Some restrictions on dietary restraint. Appetite 14, 137-141, discussion 142-143.

14. Lawson OJ, Williamson DA, Champagne CM, et al. (1995) The association of body weight, dietary intake, and energy expenditure with dietary restraint and disinhibition. Obes Res 3, $153-161$.

15. Dykes J, Brunner EJ, Martikainen PT, et al. (2004) Socioeconomic gradient in body size and obesity among women: the role of dietary restraint, disinhibition and hunger in the Whitehall II study. Int J Obes Relat Metab Disord 28, 262-268.

16. Tremblay A, Sévigny J, Leblanc C, et al. (1983) The reproducibility of a three-day dietary record. Nutr Res 3, 819-830.

17. Verdier P \& Beare-Rogers JL (1984) The Canadian Nutrient File. J Can Diet Assoc 45, 52-55.

18. Cole TJ, Bellizi MC, Flegal KM, et al. (2000) Establishing a standard definition for child overweight and obesity worldwide: international survey. BMJ 320, 1240-1243.

19. MacCallum RC, Zhang S, Preacher KJ, et al. (2002) On the practice of dichotomization of quantitative variables. Psychol Methods 7, 19-40.

20. Berkey CS \& Colditz GA (2007) Adiposity in adolescents: change in actual BMI works better than change in BMI $z$ score for longitudinal studies. Ann Epidemiol 17, 44-50.

21. Bryant EJ, King NA \& Blundell JE (2008) Disinhibition: its effects on appetite and weight regulation. Obes Rev 9, 409-419.

22. Schubert E \& Randler C (2008) Association between chronotype and the constructs of the Three-Factor-Eating Questionnaire. Appetite 51, 501-505.

23. Hays NP, Bathalon GP, McCrory MA, et al. (2002) Eating behaviour correlates of adult weight gain and obesity in healthy women aged 55-65 y. Am J Clin Nutr 75, 476-483.

24. Harden CJ, Corfe BM, Richardson JC, et al. (2009) Body mass index and age affect Three-Factor Eating Questionnaire scores in male subjects. Nutr Res 29, 379-382.

25. Lluch A, Herbeth B, Mejean L, et al. (2000) Dietary intakes, eating style and overweight in the Stanislas Family Study. Int J Obes Relat Metab Disord 24, 1493-1499.

26. de Lauzon B, Romon M, Deschamps V, et al. (2004) The Three-Factor Eating Questionnaire-R18 is able to distinguish among different eating patterns in a general population. J Nutr 134, 2372-2380.

27. Vogels N, Posthumus DL, Mariman EC, et al. (2006) Determinants of overweight in a cohort of Dutch children. Am J Clin Nutr 84, 717-724.

28. Stewart TM, Williamson DA \& White MA (2002) Rigid vs flexible dieting: association with eating disorder symptoms in nonobese women. Appetite 38, 39-44.

29. Snoek HM, van Strien T, Janssens JM, et al. (2008) Restrained eating and BMI: a longitudinal study among adolescents. Health Psychol 27, 753-759.

30. Timko CA (2007) Norms for the rigid and flexible control over eating scales in a United States population. Appetite 49, $525-528$.

31. Ackard DM, Neumark-Sztainer D, Story M, et al. (2003) Overeating among adolescents: prevalence and associations with weight-related characteristics and psychological health. Pediatrics 111, 67-74. 
32. Muise AM, Stein DG \& Arbess G (2003) Eating disorders in adolescent boys: a review of the adolescent and young adult literature. $J$ Adolesc Health 33, 427-435.

33. Goulet J, Provencher V, Piche ME, et al. (2008) Relationship between eating behaviours and food and drink consumption in healthy postmenopausal women in a real-life context. Br J Nutr 100, 910-917.

34. Provencher V, Drapeau V, Tremblay A, et al. (2004) Eating behaviours, dietary profile and body composition according to dieting history in men and women of the Québec Family Study. Br J Nutr 91, 997-1004.
35. Rennie KL, Jebb SA, Wright A, et al. (2005) Secular trends in under-reporting in young people. Br J Nutr 93, 241-247.

36. Vance VA, Woodruff SJ, McCargar LJ, et al. (2009) Self-reported dietary energy intake of normal weight, overweight and obese adolescents. Public Health Nutr 12, 222-227.

37. Babio N, Canals J, Fernandez-Ballart J, et al. (2008) Non-clinical adolescent girls at risk of eating disorder: under-reporters or restrained eaters? Nutr Hosp 23, 27-34.

38. Ventura AK, Loken E, Mitchell DC, et al. (2006) Understanding reporting bias in the dietary recall data of 11-year-old girls. Obesity (Silver Spring) 14, 1073-1084. 